\title{
BMJ Open Adult food choices in association with the local retail food environment and food access in resource-poor communities: a scoping review protocol
}

\author{
Samukelisiwe Sthokozisiwe Madlala (D) , ${ }^{1,2}$ Jillian Hill, ${ }^{1}$ Ernesta Kunneke, ${ }^{3}$ \\ Mieke Faber ${ }^{1,3}$
}

To cite: Madlala SS, Hill J, Kunneke E, et al. Adult food choices in association with the local retail food environment and food access in resourcepoor communities: a scoping review protocol. BMJ Open 2021;11:e044904. doi:10.1136/ bmjopen-2020-044904

- Prepublication history for this paper is available online. To view these files, please visit the journal online (http://dx.doi org/10.1136/bmjopen-2020044904).

Received 17 September 2020 Accepted 28 July 2021

Check for updates

(C) Author(s) (or their employer(s)) 2021. Re-use permitted under CC BY-NC. No commercial re-use. See rights and permissions. Published by BMJ.

${ }^{1}$ Non-Communicable Diseases Research Unit, South African Medical Research Council, Cape Town, South Africa

${ }^{2}$ School of Public Health,

Faculty of Community and Health Sciences, University of the Western Cape, Cape Town, South Africa

${ }^{3}$ Department of Dietetics and Nutrition, University of the Western Cape, Cape Town, South Africa

Correspondence to

Samukelisiwe Sthokozisiwe Madlala;

samukelisiwe.madlala@mrc. ac.za

\section{ABSTRACT}

Introduction The local retail food environment influences dietary patterns and food choices, as suggested in the literature. The lack of access to healthy food within this environment may result in unhealthy food choices which may lead to obesity and the development of noncommunicable diseases. Evidence suggests that resourcepoor communities may have unhealthy food environments, therefore, preventing residents from making healthy food choices. A systematic scoping review will be conducted to provide an overview of the evidence on adult food choices in association with the local retail food environment and food access in resource-poor communities.

Methods and analysis This protocol for the scoping review was developed following the Preferred Reporting Items for Systematic reviews and Meta-Analyses Extension for Scoping Reviews (PRISMA-ScR) guidelines and the framework process by Arksey and 0'Malley. Observational studies, published from July 2005 to January 2021, will be searched and screened. Keywords and medical subject headings (MeSH) terms will be used to search several multidisciplinary databases. Two independent reviewers will screen identified articles using the selection criteria and extract data using the PRISMA-ScR checklist. Descriptive numerical and thematic analysis will be performed to evaluate and categorise quantitative and qualitative data.

Ethics and dissemination Ethical approval will not be required for the review, as data from published studies will be used. The results of this scoping review will form part of a PhD thesis that will be submitted to the University of the Western Cape, South Africa. The review findings will also be presented at conferences and published in a peerreviewed journal.

Open science framework registration number https:// osf.io/shf93.

\section{INTRODUCTION}

Malnutrition in the form of overweight, obesity and underweight is the leading cause of disease globally. ${ }^{1}$ Dietary-related disease risk is determined by food choices and dietary consumption. ${ }^{2}$ Food choices are defined as foods selected and consumed based on an individual's decision which is influenced by
Strengths and limitations of this study

- The findings will provide insight on how the retail food environment plays a role in determining healthy food access and identify the barriers, enablers and mediators of food access which affect food choices of adults in resource-poor communities.

- Several multidisciplinary databases will be used in the search, as the food environment topic is extensive.

- In this systematic approach, findings from a body of knowledge that is heterogeneous in terms of methods and discipline will be summarised.

- Only studies published in English will be included.

- There will be no formal appraisal done which means possibility of inclusion of methodologically inferior studies. However, to reduce number of poor-quality studies included, only peer-reviewed and published studies will be included.

a combination of individual, environmental and economic factors. ${ }^{3}$ Food choices are also a result of the relationship between individual factors and the food environment. ${ }^{4}$ Glanz $e t$ al distinguish two types of environments that influence access to healthy food to make healthy food choices. These environments are namely the community nutrition environment (types and location of food stores and accessibility in each community), and the consumer nutrition environment (the availability of healthy and unhealthy food choices within any establishment where food is sold or served, ie, restaurant, school or work cafeteria, price, promotion and placement of food choices). ${ }^{5}$ The food environment is also referred to as the local food environment. The retail food environment combines the physical proximity to food store locations, the distribution of food stores and markets at a community level, and consumer access to healthy affordable foods at food stores or markets. ${ }^{6}$ The community and the consumer 
nutrition environment, the interest topics of this study, will be referred to as the local retail food environment.

The local retail food environment is an important determinant of food choices and may influence individual, family and population-level health. ${ }^{7}$ Furthermore, it may influence dietary patterns and food choices. ${ }^{78}$ The lack of access to healthy food within this environment may result in unhealthy food choices, which may lead to obesity and the development of non-communicable diseases (NCDs) such as cancers, cardiovascular diseases and type 2 diabetes mellitus. ${ }^{8-10}$ The local retail food environment is also a determining factor for food access. ${ }^{7}$

Food access relates to the physical and economic access to food. ${ }^{11}$ Access to food means that it must be physically procured by individuals and be economically accessible. Thus, people can afford to buy the food that is available in the local retail food environment, and in adequate amounts. ${ }^{11}$ Access to food consists of several components. Examples are quantity (sufficient amounts of food), quality (nutritionally balanced food), safety (food that is devoid of harmful substances and can impact health), and culturally acceptable and preferable foods (those that support traditional or preferred diets). ${ }^{12}$ Therefore, access to food affects food choices.

Food access in the local retail food environment is dependent on the spatial proximity of food stores, affordability, cultural appropriateness and healthiness of foods available. ${ }^{13}$ Lack of access to healthy food such as fresh fruits and vegetables is often seen in low-income communities. ${ }^{13-19}$ Communities with limited healthy foods available to residents are known as 'food desert' areas. ${ }^{20} 21$ Many resource-poor communities have a large number of fast-food restaurants, liquor stores and convenience stores supplying cheap, processed nutrient-poor foods. ${ }^{22}$ It therefore follows that people with low incomes may have poor food choices that include cheap, energynutrient dense and nutrient-deficient foods. Low-income individuals living in food deserts are at a greater risk of developing NCDs in comparison to individuals in highresource communities. ${ }^{17} 18202324$ Increasing access to affordable and healthy food in resource-poor communities is therefore important.

\section{STUDY RATIONALE}

The rise in interest in the food environment can be attributed to the demand to improve dietary, nutritional and health outcomes. ${ }^{25}$ The food environment is an important approach for implementing interventions that support healthy diets and address malnutrition as this is where consumers make decisions on what food to buy and consume. ${ }^{26}$ Retail food environments influence the type of food purchased and consumed. ${ }^{5}$ The accessibility of healthy food in the retail food environment enables people to have better quality diets with fruit and vegetables, and therefore better health outcomes. There are many intervention strategies used to improve access to food in urban and rural communities; these include increasing the number of chain supermarkets in food deserts, increasing the number and supporting farmers markets, establishing community gardens, increasing the price of unhealthy food and serving healthier convenience foods. ${ }^{13212728}$

While there are interventions to improve access to food in urban and rural communities, many people are still struggling to purchase and consume healthy food. ${ }^{13} 212728$ Healthy food access is important for enhancing the economy and improving community health. To address the healthy food access issue in communities, it is necessary understanding the role of the local retail food environment in enabling or hindering resource-poor community residents' access to healthy food for making better food choices. Past reviews conducted on the food environment have focused on associations between school food environments and children's diet ${ }^{29}{ }^{30}$ child weight status, ${ }^{31}$ food environment in high-income countries ${ }^{32}$ and low-income and middleincome countries..$^{25}$ The majority of literature to date has also focused on the food environment and overweight/ obesity and physical activity and not given much attention to dietary outcomes more especially food choices. To our knowledge, this will be the first review to examine the association of the local retail food environment and food access on the food choices of adults. It is important to understand the relationship between the local retail food environment and food access and adult food choices so that appropriate interventions can be created to prevent NCDs in adult population residing in resource-poor communitites. The aim of the scoping review is to gain an understanding of what is the association between adult food choices and the factors that determine healthy food access in the local retail food environment of resourcepoor communities.

The objectives are to:

1. assess whether adult food choices are associated with the local retail food environment in resource-poor communities; and

2. determine the barriers and facilitators for healthy food access in resource-poor communities.

\section{METHODS AND ANALYSIS}

\section{Protocol structure}

The protocol was developed following the framework described by Arksey and O'Malley. ${ }^{33}$ The framework includes five stages namely (1) identifying the research question; (2) identifying relevant studies; (3) study selection; (4) charting the data and (5) collating, summarising and reporting the results. ${ }^{33}$ The final protocol was registered with the Open Science Framework on 9 September 2020 (https://osf.io/shf93).

\section{Step 1: identifying research questions}

The population, concept and context (PCC) search strategy was used for the development of the research questions. ${ }^{34}$ This search strategy will enable the 
Table 1 Literature search strategy

\begin{tabular}{ll}
\hline Concept & MeSH terms/Keywords \\
\hline Diet/food choice & $\begin{array}{l}\text { MeSH terms: Diet, healthy OR Diet western OR Diet high fat } \\
\text { Keywords: Food choice OR food behaviours OR adult OR food OR fruit OR vegetable OR nutrition OR } \\
\text { processed food OR salty food OR fatty foods OR sugar-sweetened beverages OR fast food OR street } \\
\text { food. } \\
\text { Keywords: Food environment OR nutrition environment OR Local retail food environment OR } \\
\text { neighbourhood OR consumer nutrition environment OR community nutrition environment OR food desert } \\
\text { OR food swamp }\end{array}$ \\
Resource poor & $\begin{array}{l}\text { MeSH terms: Low income OR low-income population OR poverty. } \\
\text { Keywords: Low income OR low socio-economic status OR disadvantaged OR resource poor OR poor OR } \\
\text { deprived }\end{array}$ \\
\hline Food access & $\begin{array}{l}\text { MeSH terms: Food deserts OR Food security. } \\
\text { quality } \\
\text { Keywords: Food store OR supermarket OR grocery store OR convenience store OR corner store OR fast } \\
\text { food OR restaurant OR street vendor }\end{array}$ \\
\hline
\end{tabular}

$\mathrm{MeSH}$, medical subject headings.

identification of relevant studies to meet the aim of the scoping review. ${ }^{35}$ For this scoping review, the population is male and female adults, the concept is food choices and the context is the local retail food environment and food access in resource-poor settings. To understand the association between food choices and the food environment and food access, the following research questions will be used to guide the search strategy.

- What is the association between adult food choices and the local retail food environment in resourcepoor communities?

- Does food accessible in the local retail food environment influence healthy food choices?

- What characteristics of the local retail food environment enable food access or limit food access?

\section{Step 2: identifying relevant studies}

A search on published literature will be conducted using the following databases, PubMed/Medline, CINAHL, EBSCOhost, Green FILE, PsycARTICLES, Social Science
Research Network, Scopus, Science Direct and Web of Science. Table 1 presents a summary of the search keywords or medical subject headings (MeSH) terms that will be used. The Boolean (AND, OR) method will be used to combine search terms. The original search strategy was developed in PubMed and will be adapted to the other databases. The PubMed search strategy is presented in table 2. A reference list of bibliographies of studies found will be checked for additional sources.

\section{Step 3: study selection}

Eligibility criteria will be used to ensure that the studies included in the scoping review are relevant to the research questions.

\section{Inclusion criteria}

- Observational studies (ie, cohort, cross-sectional, case-control and ecological studies) reporting on the association between adult food choices (outcome)

Table 2 Electronic search record of PubMed database

\begin{tabular}{|c|c|c|c|}
\hline Date & Keyword searched & $\begin{array}{l}\text { Database } \\
\text { used }\end{array}$ & $\begin{array}{l}\text { Number of } \\
\text { publications retrieved }\end{array}$ \\
\hline $\begin{array}{l}02 \text { February } \\
2021\end{array}$ & $\begin{array}{l}\text { (/(Food choice[Title/Abstract] OR food behaviours[Title/Abstract] OR adult[Title/Abstract] OR } \\
\text { food[Title/Abstract] OR fruit[Title/Abstract] OR vegetable[Title/Abstract] OR diet[Title/Abstract] OR } \\
\text { nutrition[Title/Abstract] OR processed food[Title/Abstract] OR salty food[Title/Abstract] OR fatty } \\
\text { foods[Title/Abstract] OR sugar-sweetened beverages[Title/Abstract] OR fast food[Title/Abstract] OR } \\
\text { street food.[Title/Abstract]) AND (Food environment[Title/Abstract] OR nutrition environment[Title/ } \\
\text { Abstract] OR Local retail food environment[Title/Abstract] OR neighbourhood[Title/Abstract] OR } \\
\text { consumer nutrition environment[Title/Abstract] OR community nutrition environment[Title/Abstract] } \\
\text { OR food desert[Title/Abstract] OR food swamp[Title/Abstract])) AND (Low income[Title/Abstract] } \\
\text { OR low socio-economic status[Title/Abstract] OR disadvantaged[Title/Abstract] OR resource } \\
\text { poor[Title/Abstract] OR poor[Title/Abstract] OR poverty[Title/Abstract] OR deprived[Title/Abstract])) } \\
\text { AND (Food access[Title/Abstract] OR food availability[Title/Abstract] OR food cost[Title/Abstract] } \\
\text { OR food affordability[Title/Abstract] OR food price[Title/Abstract] OR food quality[Title/Abstract])) } \\
\text { AND (Food store[Title/Abstract] OR supermarket[Title/Abstract] OR grocery store[Title/Abstract] OR } \\
\text { convenience store[Title/Abstract] OR corner store[Title/Abstract] OR fast food[Title/Abstract] OR } \\
\text { restaurant[Title/Abstract] OR street vendor[Title/Abstract]) } \\
\text { Filters applied: Results by year 2005-2021 }\end{array}$ & PubMed & 69 \\
\hline
\end{tabular}


and the local retail food environment and food access (exposures) in resource-poor communities.

- Empirical and theoretical studies.

- Studies including adults 18-65 years old.

- Studies on the food environment outside the home environment but within the retail food environment, which is the community and the consumer food environment.

- Studies on food access, food choices and diets of adults in resource-poor communities.

- English peer-reviewed journal articles from July 2005 to January 2021.

\section{Exclusion criteria}

- Experimental studies (randomised controlled trials), systematic reviews and meta-analysis.

- Research not reported in peer-reviewed journals, studies discussing organisational food environment (home, school and work) and information environment (television advertising).

- Studies on children, pregnant women and the elderly.

- Studies that only focus on the food environment and nutritional status.

- Studies that focus on indirect measures of diet, such as food purchasing or the number of trips to food stores.

- Papers written in another language besides English and research papers published before July 2005 will be excluded from the study.

Eligible articles will be uploaded into EndnoteX9 library, and duplicates identified and removed. Two levels will be followed when screening articles. Level one involves two reviewers screening the title and abstracts (TIABS) of searched articles to identify eligible ones. In level two, the two reviewers will read the full-text articles to determine whether they meet the eligibility criteria. Both levels of screening will be performed on the Rayyan Qatar Computing Research Institute (QCRI) systematic reviews web application. ${ }^{36} \mathrm{~A}$ third reviewer will be consulted should there be any disagreement on full-text articles to reach a consensus. The Preferred Reporting Items for Systematic Reviews and Meta-Analyses extension for Scoping Reviews (PRISMA-ScR) checklist will be used to guide the selection process. ${ }^{37}$ The study selection process is presented in the PRISMA flow diagram (see figure 1). ${ }^{38}$

\section{Step 4: charting the data}

The PCC format will be used to guide the data extraction. A data charting form, as per the framework of Arksey and O'Malley, ${ }^{33}$ will be developed to extract data from studies included (see table 3). The data extraction form will be piloted by two reviewers on $10 \%$ of the sample of included studies. ${ }^{39}$ This will be done to ensure that reviewers understand the data collection procedure and whether all relevant information is correctly captured. The data extraction form will be revised should the reviewers decide that relevant items are not adequately captured. Inter-rater reliability will be attained by comparing $20 \%$

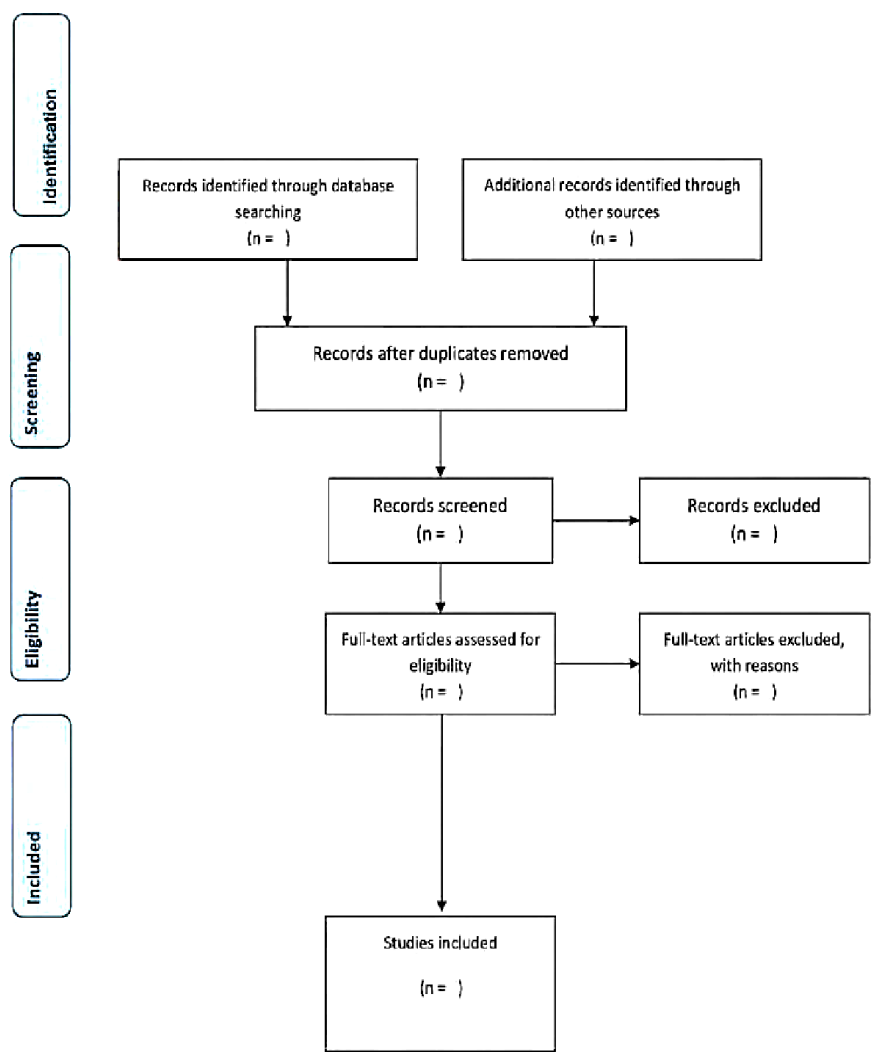

Figure 1 Preferred Reporting Items for Systematic Reviews and Meta-Analysis flow diagram for the scoping review process.

of the sample of independently screened papers by the two reviewers. ${ }^{39}$ Disagreements will be discussed by the two reviewers to reach consensus or through consulting a third reviewer.

\section{Reducing bias}

Eligibility criteria will be used to reduce selection bias. Two reviewers will review eligible studies and this will reduce error and increase reliability of the findings of the scoping review. Methods to reduce bias are presented in table 4 . A systematic approach will be followed when reviewing the research evidence to ensure the relevance and validity of results. By including different types of evidence or data sources, such as quantitative or qualitative research, expert opinion and policy documents, heterogeneity will be ensured. ${ }^{35}$

\section{Step 5: collating, summarising and reporting results}

The process of collating, summarising and reporting results will follow three steps as recommended by Levac et $a l .{ }^{35}$ In the first step, a descriptive numerical summary for quantitative studies and thematic analysis for qualitative studies will be done. The descriptive numerical summary will state the number of studies included, types of study design, year of publication, characteristics of populations and the countries where the studies were done. With regards to the qualitative analysis, descriptive themes will be developed by categorising ideas by topic/concept. In the second step, the results and outcome of the study 
Table 3 Data extraction form

1. Authors

2. Title of study

3. Year of publication

4. Aim/objective of the study

5.Study setting (location/

country)

6. Study participants

(number, age, gender and

ethnicity)

\section{Sampling method}

$\begin{array}{llr}\begin{array}{l}\text { 8. Study design/publication } \\ \text { type }\end{array} & \text { Cross-sectional: } \square & \text { Cohort } \\ & \text { Case-control } \square & \text { Others } \\ \text { 9. Data collection method } & \text { Quantitative } \square & \text { Mixed method } \\ & \text { Qualitative } \\ \end{array}$

10. Data analysis

11. Reported outcomes

Study findings relevant to study objectives.

12. Most relative findings Findings as relates to food choices and healthy diet measured by fruit and vegetable intake, various food group intake, intake of salty and fatty foods, sugar-sweetened beverage intake, fast-food intake, diet quality, energy and micronutrient intake and healthy diet score vs unhealthy diet scores. ${ }^{30}$

13. Facilitators

Describe the factors that enable healthy food choices and food access in the local retail food environment.

14. Barriers

Describe the factors that hinder healthy food choices and food access in the local retail food environment.

in relation to the aim of the research question will be discussed. The third step involves reporting the implications of the findings in terms of future research, practice and policy. ${ }^{34}$

Table 4 Types of bias and resolution

\begin{tabular}{ll}
\hline Bias & Resolution \\
\hline Selection bias & Clear definition of exposure and \\
& outcomes in the inclusion and exclusion \\
& criteria. \\
& Two reviewers will independently screen \\
& TIABS and full text articles and extract \\
& data to reduce bias. \\
& Inter-rater reliability will be assessed to \\
& reduce bias. \\
& The Rayyan software will be used for \\
& screening TIABS and full text articles. \\
& This software allows for 'blind screening' \\
& among reviewers, this will reduce bias. \\
Publishing & $\begin{array}{l}\text { All research findings whether positive or } \\
\text { negative will be reported in the findings. }\end{array}$ \\
bias & Only English articles were selected. \\
Language bias & Literature states that excluding non-English \\
studies does not impact outcomes of most \\
reviews.
\end{tabular}

\section{Patient and public involvement}

There was no patient or public involvement in the design of this protocol.

\section{ETHICS AND DISSEMINATION}

Ethical approval will not be required for the review, as data from published studies will be used for the analysis. The results of this scoping review will form part of a $\mathrm{PhD}$ thesis that will be submitted to the University of the Western Cape. The review findings will also be presented at conferences and published in a peer-reviewed journal.

Contributors SSM and MF conceived the idea and developed the research questions and methods for the protocol. SSM was responsible for drafting the manuscript. MF supervised the writing of the protocol. MF, JH and EK critically revised the manuscript for its methodological and scientific content. All authors approved the final version of the manuscript.

Funding The work reported herein was made possible through funding by the South African Medical Research Council through its Division of Research Capacity Development under the Internship Scholarship Programme from funding received from the South African National Treasury (grant/award number :N/A).

Disclaimer The views expressed are those of the authors and not necessarily those of the South African Medical Research Council.

Competing interests None declared.

Patient and public involvement Patients and/or the public were not involved in the design, or conduct, or reporting, or dissemination plans of this research. 
Patient consent for publication Not required.

Provenance and peer review Not commissioned; externally peer reviewed.

Open access This is an open access article distributed in accordance with the Creative Commons Attribution Non Commercial (CC BY-NC 4.0) license, which permits others to distribute, remix, adapt, build upon this work non-commercially, and license their derivative works on different terms, provided the original work is properly cited, appropriate credit is given, any changes made indicated, and the use is non-commercial. See: http://creativecommons.org/licenses/by-nc/4.0/.

\section{ORCID iD}

Samukelisiwe Sthokozisiwe Madlala http://orcid.org/0000-0002-7715-2147

\section{REFERENCES}

1 Swinburn BA, Kraak VI, Allender S, et al. The global syndemic of obesity, undernutrition, and climate change: the Lancet Commission report. Lancet 2019;393:791-846.

2 Lytle L, Myers A. Measures registry user guide: food environment. Washington (DC): National Collaborative on Childhood Obesity Research, 2017. http://nccor.org/tools-mruserguides/wp content/ uploads/2017/NCCOR_MR_User_Guide_Food_Environment-FINAL. pdf

3 Buttriss J, Stanner S, McKevith B, et al. Successful ways to modify food choice: lessons from the literature. Nutr Bulletin 2004;29:333-43.

4 Herforth A, Ahmed S. The food environment, its effects on dietary consumption, and potential for measurement within agriculturenutrition interventions. Food Security 2015;7:505-20.

5 Glanz K, Sallis JF, Saelens BE, et al. Healthy nutrition environments: concepts and measures. Am J Health Promot 2005;19:330-3.

6 Centers for Disease Control and Prevention (CDC). General food environment resources, 2014. Available: http://www.cdc.gov/ healthyplaces/healthtopics/healthyfood/general.htm

7 Rose D. Access to healthy food: a key focus for research on domestic food insecurity. J Nutr 2010;140:1167-9.

8 Black C, Moon G, Baird J. Dietary inequalities: what is the evidence for the effect of the neighbourhood food environment? Health Place 2014;27:229-42.

9 Story M, Kaphingst KM, Robinson-O'Brien R, et al. Creating healthy food and eating environments: policy and environmental approaches. Annu Rev Public Health 2008;29:253-72.

10 Giskes K, Kamphuis CBM, van Lenthe FJ, et al. A systematic review of associations between environmental factors, energy and fat intakes among adults: is there evidence for environments that encourage obesogenic dietary intakes? Public Health Nutr 2007;10:1005-17.

11 Food and Agriculture Organization (FAO). An introduction to the basic concepts of food security, 2008. Available: http://www.fao.org/3/aal936e.pdf

12 Leroy JL, Ruel M, Frongillo EA, et al. Measuring the food access dimension of food security: a critical review and mapping of indicators. Food Nutr Bull 2015;36:167-95.

13 Evans A, Banks K, Jennings R, et al. Increasing access to healthful foods: a qualitative study with residents of low-income communities. Int J Behav Nutr Phys Act 2015;12 Suppl 1:S5.

14 Kim M, Budd N, Batorsky B, et al. Barriers to and facilitators of stocking healthy food options: viewpoints of Baltimore City small storeowners. Ecol Food Nutr 2017;56:17-30.

15 Grimm KA, Moore LV, Scanlon KS, et al. Access to healthier food retailers - United States, 2011. MMWR Suppl 2013;62:20-6.

16 Beaulac J, Kristjansson E, Cummins S. A systematic review of food deserts, 1966-2007. Prev Chronic Dis 2009;6:A105.
17 Larson N, Story M. A review of environmental influences on food choices. Ann Behav Med 2009;38 Suppl 1:56-73.

18 Morland KB, Evenson KR. Obesity prevalence and the local food environment. Health Place 2009:15:491-5.

19 Powell LM, Slater S, Mirtcheva D, et al. Food store availability and neighborhood characteristics in the United States. Prev Med 2007:44:189-95.

20 Zenk SN, Mentz G, Schulz AJ, et al. Longitudinal associations between observed and perceived neighborhood food availability and body mass index in a multiethnic urban sample. Health Educ Behav 2017;44:41-51.

21 Kumar S, Quinn SC, Kriska AM, et al. "Food is directed to the area": African Americans' perceptions of the neighborhood nutrition environment in Pittsburgh. Health Place 2011;17:370-8.

22 Bell J, Mora G, Hagan E. Access to healthy food and why it matters: a research review. PolicyLink, The Food Trust: Oakland, 2013.

23 Shih M, Dumke KA, Goran Ml, et al. The association between community-level economic hardship and childhood obesity prevalence in Los Angeles. Pediatr Obes 2013;8:411-7.

24 Kwate NOA, Yau C-Y, Loh J-M, et al. Inequality in obesigenic environments: fast food density in New York City. Health Place 2009;15:364-73.

25 Turner C, Kalamatianou S, Drewnowski A. Erratum to Turner et al. food environment research in low- and middle-income countries: a systematic scoping review. Adv Nutr 2019;10:387-97.

26 Downs SM, Ahmed S, Fanzo J, et al. Food environment typology: advancing an expanded definition, framework, and methodological approach for improved characterization of wild, cultivated, and built food environments toward sustainable diets. Foods 2020;9:532.

27 Keener D, Goodman K, Lowry A. Recommended community strategies and measurements to prevent obesity in the United States: implementation and measurement guide. Atlanta: U.S. Department of Health and Human Services, Centers for Disease Control and Prevention, 2009

28 Giang T, Karpyn A, Laurison HB, et al. Closing the grocery gap in underserved communities: the creation of the Pennsylvania fresh food financing initiative. J Public Health Manag Pract 2008;14:272-9.

29 Williams J, Scarborough P, Matthews A, et al. A systematic review of the influence of the retail food environment around schools on obesity-related outcomes. Obes Rev 2014;15:359-74.

30 Engler-Stringer R, Le H, Gerrard A, et al. The community and consumer food environment and children's diet: a systematic review. BMC Public Health 2014;14:522.

31 da Costa Peres CM, Gardone DS, Costa BVdeL, et al. Retail food environment around schools and overweight: a systematic review. Nutr Rev 2020;78:841-56.

32 Kenny T-A, Little M, Lemieux T, et al. The retail food sector and Indigenous peoples in high-income countries: a systematic scoping review. Int J Environ Res Public Health 2020;17:8818.

33 Arksey H, O'Malley L. Scoping studies: towards a methodological framework. Int J Soc Res Methodol 2005;8:19-32.

34 Peters MDJ. In no uncertain terms: the importance of a defined objective in scoping reviews. JBI Database System Rev Implement Rep 2016;14:1-4.

35 Levac D, Colquhoun H, O'Brien KK. Scoping studies: advancing the methodology. Implement Sci 2010;5:1-9.

36 Ouzzani M, Hammady H, Fedorowicz Z, et al. Rayyan-a web and mobile APP for systematic reviews. Syst Rev 2016;5:210.

37 Tricco AC, Lillie E, Zarin W, et al. PRISMA extension for scoping reviews (PRISMA-ScR): checklist and explanation. Ann Intern Med 2018;169:467-73.

38 Peters M, Godfrey C, Mclnerney P. Methodology for JBI Scoping Reviews. In: Aromataris E, ed. The Joanna Briggs Institute reviewers manual, 2015: 3-24.

39 Bussiek P-BV, De Poli C, Bevan G. A scoping review protocol to map the evidence on interventions to prevent overweight and obesity in children. BMJ Open 2018;8:e019311. 